\title{
Revisión sistemática y evidencia sobre exposición profesional a ruido y efectos extra-auditivos de naturaleza cardiovascular
}

\author{
Systematic review and evidence on occupational \\ noise exposure and extra-auditory effects of \\ cardiovascular nature
}

\section{Rosana Cortés Barragán}

Escuela Nacional de Medicina del Trabajo, Instituto de Salud Carlos III, Madrid, España.

Jerónimo Maqueda Blasco

Escuela Nacional de Medicina del Trabajo, Instituto de Salud Carlos III, Madrid, España.

Elena Ordaz Castillo

Escuela Nacional de Medicina del Trabajo, Instituto de Salud Carlos III, Madrid, España.

Ángel Asúnsolo del Barco

Departamento de Ciencias Médico Sociales, Universidad de Alcalá, Alcalá de Henares, Madrid, España.

Agustín Silva Mato

Departamento de Ciencias Médico Sociales, Universidad de Alcalá, Alcalá de Henares, Madrid, España.

\section{Eva Bermejo García}

Escuela Nacional de Medicina del Trabajo, Instituto de Salud Carlos III, Madrid, España.

María Fe Gamo González

Escuela Nacional de Medicina del Trabajo, Instituto de Salud Carlos III, Madrid, España.

Correspondencia:

Jerónimo Maqueda Blasco

Director de la Escuela Nacional de Medicina del Trabajo

Pabellón n 8, Ciudad Universitaria

28040 Madrid (España)

Tfno: 918224013

jmaqueda@isciii.es

Proyecto financiado por el Instituto de Salud Carlos III, PI07/90034

Resumen

Trabajo de revisión que tiene como objetivo conocer el nivel de evidencia existente sobre los efectos cardiovasculares debidos a la exposición profesional al ruido, mediante el análisis sistemático de la producción científica publicada entre 1995 y 2008. La búsqueda de las referencias bibliográficas se realizó mediante términos "Major Topic" y "MeSH" para MEDLINE y términos "Decriptors" para OSH update. Se recuperaron un total de 236 referencias y se incluyeron en la revisión todos los estudios experimentales, estudios de cohortes y estudios casos control y aquellos estudios trasversales que cumplieron, al menos, con cinco de los nueve criterios que la declaración STROBE establece para los estudios observacionales. Fueron seleccionados para su revisión a texto completo un total de 23 artículos: 12 estudios transversales, 7 estudios de cohortes, dos estudios casos control, 1 meta-análisis y 1 estudio experimental.

La asignación del nivel de evidencia se realizó de acuerdo a los criterios SIGN. La selección y revisión de los artículos se realizó mediante revisión por pares, los casos de discrepancia entre pares se resolvió mediante consenso. 
En relación con los efectos cardiovasculares de la exposición laboral a ruido, nuestra revisión encuentra un mayor nivel de evidencia (1++) para el incremento de la tensión arterial e incremento de la frecuencia cardiaca. Estudios con nivel de evidencia 2++ muestran resultados en esa misma dirección.

La enfermedad isquémica, infarto agudo de miocardio y coronariopatía muestran un nivel de evidencia 2++.

La alteraciones metabólicas de lípidos y alteraciones de parámetros vasculares disponen de un nivel bajo de evidencia ${ }^{3}$.

El control del ruido en entornos laborales es un factor de prevención del riesgo cardio-vascular. HTA, riesgo coronario y otras enfermedades cardiovasculares deben considerarse en los protocolos de vigilancia médica específica de los trabajadores expuestos a ruido.

(Med Segur Trab 2009; 55 (215): 28-51)

Palabras clave: Ruido, salud laboral, enfermedad cardiovascular, hipertensión arterial.

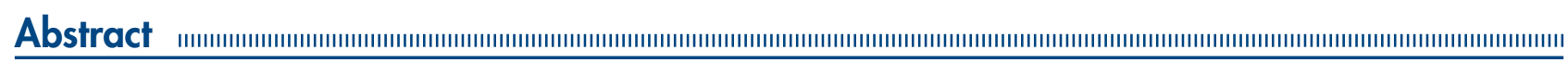

Review aimed to identify the level of scientific evidence about the relationship between cardiovascular diseases and occupational noise exposure, by a systematic analysis of the scientific literature published between 1995 and 2008. "Major Topic" and "MeSH" terms for MEDLINE and "Decriptors" for OSH update was used for the search of references. We retrieved a total of 236 references. Experimental studies, cohort studies and case control studies were all included in the review and those cross-sectional studies with at least five of the nine criteria from the STROBE declaration provides for observational studies. 23 articles were selected for full text review: 12 cross-sectional studies, 7 cohort studies, two case control studies, 1 meta-analysis and 1 experimental study.

The evidence level was performed according to SIGN criteria. The selection and review of articles was done by peer review, cases of disagreement between pairs was resolved by consensus.

Our review found a higher level of evidence (1++) for increasing blood pressure and increased heart rate and occupational noise exposure. Studies with a level of evidence $2++$ show results in the same direction.

Ischemic heart disease, myocardial infarction and coronary diseases show a level of evidence 2++.

Metabolic changes of lipid parameters and vascular abnormalities have a low level of evidence ${ }^{3}$.

Noise control in working environments is a measure for cardiovascular risk prevention. Hypertension, coronary risk and other cardiovascular diseases should be considered in medical surveillance practice of workers exposed to noise.

(Med Segur Trab 2009; 55 (215): 28-51)

Keywords: Noise, occupational health, cardiovascular disease, blood pressure. 


\section{INTRODUCCIÓN}

El ruido puede definirse como un sonido no agradable o conjunto de sonidos no coordinados que originan sensaciones desagradables e interfieren con la actividad humana, siendo el ruido una apreciación subjetiva y molesta del sonido.

El ruido es uno de los agentes físicos más extendidos en el medio laboral, según datos de la tercera encuesta europea de condiciones de trabajo, en el año 2000 un $20 \%$ de los trabajadores europeos estaban expuestos a ruidos elevados en el entorno de trabajo ${ }^{1}$, prevalencia que en la cuarta edición de esta encuesta, que incluye ya la ampliación de la Unión Europea, asciende a un $30 \%^{2}$.

En nuestro país la prevalencia de trabajadores que soportan un ruido molesto asciende a un 37\% según la VI Encuesta Nacional de Condiciones de Trabajo (VI ENCT) 3 .

Estos indicadores de prevalencia ponen de manifiesto que, por su extensión, el ruido sigue siendo un problema actual en el ámbito de la mejora de la calidad de vida y condiciones de trabajo.

La legislación en materia de protección de los trabajadores contra el ruido orienta la acción preventiva a la vigilancia y control de los efectos auditivos derivados de la exposición laboral al ruido ${ }^{4}$, sin embargo existen exposiciones laborales a niveles de presión sonora insuficiente para producir pérdidas auditivas pero capaces de provocar efectos en otros órganos, interferir en la comunicación y en el desempeño de tareas 5 .

Definimos los efectos extra auditivos como todos aquellos efectos que afectan a la salud y al bienestar del sujeto y que son causados por exposición al ruido con exclusión de los efectos producidos directamente sobre el aparato auditivo o sobre la audición ${ }^{6}$.

El ruido, por tanto, puede provocar malestar, disminuir o impedir la atención, alterar: la capacidad de concentración, el sueño y el rendimiento e inducir comportamientos psicológicos alterados, incrementar el riesgo de accidentes de trabajo, y provocar alteraciones fisiológicas en el sistema cardiovascular, entre otros ${ }^{7}$.

Según la Organización Mundial de la Salud (OMS) solamente se ha encontrado evidencia significativa para efectos extra-auditivos relacionados con la hipertensión arterial (HTA) ${ }^{8}$.

Las enfermedades cardiovasculares (ECV) constituyen un problema de salud de primer orden. Según datos de la Organización Mundial de la Salud en el año 2003, se produjeron 16,7 millones de muertes en todo el mundo, debido a ECV y más de una tercera parte de éstas defunciones se dieron en adultos de mediana edad ${ }^{9}$. En Europa las ECV son la primera causa de muerte, tienen una alta incidencia, son una de las principales causas de discapacidad y repercuten seriamente en los costes de la asistencia sanitaria.

En España los datos sobre mortalidad indican que las ECV fueron la primera causa de muerte en 2006 (32,5\% del total de defunciones) porcentaje superior al de las muertes por cáncer o por otras causas ${ }^{10}$.

Estudios epidemiológicos sugieren que el ruido puede ser un factor de riesgo cardiovascular, como consecuencia de mecanismos de producción mediados por una reacción de stress, que probablemente desecancadena entre otras cosas: una respuesta del Sistema Nervioso Autónomo con una activación del sistema simpático y sistema neuroendocrino, aumento de cortisol, aumento de grasa visceral y aumento de resistencia a Insulina ${ }^{11 ; 12}$.

La mayor evidencia se ha encontrado en la relación entre exposición laboral a ruido con modificaciones de la tensión arterial por encima de umbrales de ruido entre 55-116 dbA ${ }^{13 ; 14}$.

En esta revisión se realiza un análisis sistemático de la literatura publicada en el periodo 1995-2008 sobre efectos cardiovasculares por exposición laboral al ruido en 
población trabajadora, tiene como antecedentes dos revisiones previas: La primera revisión se llevó a cabo por Smith \& Broadbent en 1989 y publicada en $1992^{6}$. Posteriormente, en 1999 Butler M.P, \& col publica una nueva revisión sobre los efectos extra-auditivos del ruido de los trabajos publicados entre 1988 y $1995^{15}$.

En nuestra revisión, hemos tomado como referencia estos dos trabajos, a los que hemos añadido los resultados sobre los niveles de evidencia científica del Scottish Intercollegiate Guidelines Network (SIGN) ${ }^{16}$, al objeto tanto de encontrar posibles nuevos hallazgos de la producción científica publicada entre 1995 y 2008 y ver si el grado de evidencia ha mejorado en estos últimos catorce años.

\section{OBJETIVOS}

Este estudio pretende alcanzar dos objetivos. El primero de ellos, el de disponer de un conocimiento sobre la evidencia existente entre exposición laboral al ruido y efectos cardiovasculares aparecida en la producción científica en el periodo comprendido entre 1995 y 2008.

El segundo objetivo, se dirige a caracterizar la producción científica en relación a este tema en lo que se refiere al tipo de diseños empleados y calidad metodológica de los estudios.

\section{MATERIAL Y MÉTODOS}

Como fuentes de información se utilizaron las bases de datos bibliográficas MEDLINE y OSH update y se definió una estrategia de búsqueda utilizando, para MEDLINE los términos "Major Topic" y "MeSH" y los términos "Decriptors" para OSH update (tabla 1).

Tabla 1: Estrategia de búsqueda en MEDLINE y OSH UPDATE

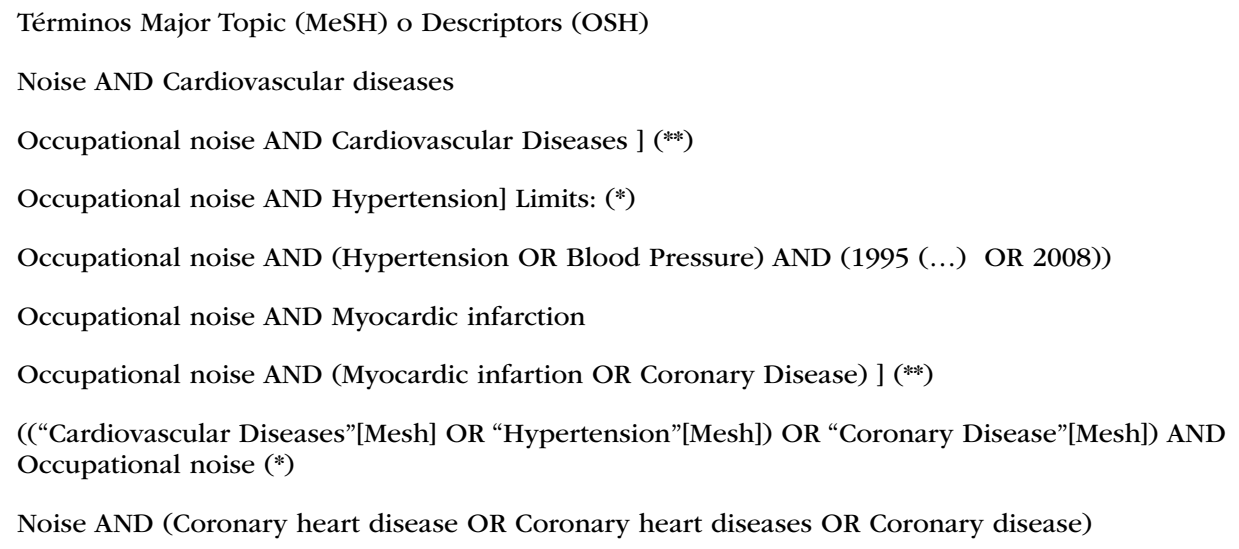

(*) Limits: Publication Date from 1995/01/01 to 1998/12/31

(**) Limits: Publication Date from $1999 / 01 / 01$ to $2008 / 12 / 31$

(***) Limits: Publication Date from 1995/01/01 to 2008/12/31

Se incluyeron para su revisión a texto completo aquellos artículos obtenidos a partir de la estrategia de búsqueda que cumplían los siguientes criterios:

1. Año de publicación entre 1995 y 2008.

2. El estudio debía cuantificar, medir o describir la relación entre el ruido y el efecto extra auditivo analizado.

3. Ensayo, estudios experimentales, meta-análisis, estudios de cohorte y estudios casos control. 
4. Estudios observacionales transversales que cumplieran los requisitos de calidad metodológica de la Iniciativa STROBE.

5. Estudios publicados en inglés o español.

La calidad de los estudios transversales se evaluó según la declaración de la Iniciativa STROBE para la comunicación de estudios observacionales. Incluyéndose en la revisión aquellos que cumplieron cinco o más puntos esenciales de los nueve criterios, que para el apartado "método" describe la declaración ${ }^{17}$.

El análisis sistemático de los artículos seleccionados incluyó, además de la valoración de la calidad metodológica, siguiendo criterios acordes con los criterios de Sackett et al y Jadad $^{18-23}$, los aspectos reflejados en la tabla 2.

Tabla 2: Aspectos del análisis sistemático de los artículos

\title{
CARACTERÍSTICAS ESTUDIADAS
}

\author{
Tipo de diseño \\ Población y muestra \\ Variables de efecto y métodos de evaluación \\ Variables factores y métodos de evaluación \\ Criterios de clasificación \\ Factores de confusión \\ Técnicas de análisis estadístico \\ Técnicas de análisis estadístico \\ Resultados \\ Conclusiones \\ Nivel de evidencia
}

A cada uno de los efectos estudiados en los artículos revisados se les asignó un nivel de evidencia científica de acuerdo a los criterios del Scottish Intercollegiate Guidelines Network (SIGN) (tabla 3) ${ }^{16}$ : 
Tabla 3: Criterios de evidencia científica y grados de recomendación (SIGN)

\begin{tabular}{|c|c|c|c|}
\hline $\begin{array}{l}\text { Grado de } \\
\text { evidencia }\end{array}$ & Criterios de evidencia & $\begin{array}{l}\text { Criterios para } \\
\text { recomendación }\end{array}$ & $\begin{array}{l}\text { Grado de } \\
\text { recomendación }\end{array}$ \\
\hline $1++$ & $\begin{array}{l}\text { Metanálisis, revisiones sistemáticas } \\
\text { de estudios aleatorizados } \\
\text { y controlados (EAC) o EAC de alta } \\
\text { calidad y con muy bajo riesgo } \\
\text { de sesgo }\end{array}$ & $\begin{array}{l}\text { Al menos, un metanálisis, } \\
\text { una revisión sistemática } \\
\text { o un EAC clasificado como 1++ } \\
\text { y directamente aplicable a la } \\
\text { población diana de la GPC } \\
\text { o una revisión sistemática de EAC } \\
\text { o un conjunto de evidencia constituido } \\
\text { principalmente por estudios } \\
\text { clasificados como } 1+\text { directamente } \\
\text { aplicables a la población diana } \\
\text { de la GPC y que muestran coherencia } \\
\text { en sus resultados. }\end{array}$ & A \\
\hline $1+$ & $\begin{array}{l}\text { Metanálisis, revisiones sistemáticas } \\
\text { de EAC o EAC bien desarrollados } \\
\text { y con bajo riesgo de sesgo. }\end{array}$ & $\begin{array}{l}\text { Un conjunto de evidencia } \\
\text { constituido por estudios clasificados } \\
\text { como } 2++ \text { directamente aplicables } \\
\text { a la población diana de la GPC y }\end{array}$ & $\mathrm{B}$ \\
\hline $1-$ & $\begin{array}{l}\text { Metanálisis, revisiones sistemáticas } \\
\text { de EAC o EAC con alto riesgo de } \\
\text { sesgo. }\end{array}$ & $\begin{array}{l}\text { que muestran coherencia en sus } \\
\text { resultados, o evidencia extrapolada } \\
\text { a partir de estudios clasificados } \\
\text { como } 1++ \text { o } 1+\text {. }\end{array}$ & \\
\hline $2++$ & $\begin{array}{l}\text { Revisiones sistemáticas de } \\
\text { estudios de casos y controles o } \\
\text { de estudios de cohortes de alta } \\
\text { calidad o estudios de casos y } \\
\text { controles, y estudios de cohortes } \\
\text { con muy bajo riesgo de sesgo y } \\
\text { con elevada probabilidad de que } \\
\text { la relación sea causal. }\end{array}$ & $\begin{array}{l}\text { Un conjunto de evidencia } \\
\text { constituido por estudios clasificados } \\
\text { como } 2+\text { directamente aplicables } \\
\text { a la población diana de la GPC y } \\
\text { que muestran coherencia en sus } \\
\text { resultados, o evidencia extrapolada } \\
\text { a partir de estudios clasificados } \\
\text { como } 2++.\end{array}$ & $\mathrm{C}$ \\
\hline $2+$ & $\begin{array}{l}\text { Estudios de casos y controles } \\
\text { y estudios de cohortes bien } \\
\text { desarrollados, con bajo riesgo } \\
\text { de sesgo y con probabilidad } \\
\text { moderada de que la relación } \\
\text { sea causal. }\end{array}$ & & \\
\hline $2-$ & $\begin{array}{l}\text { Estudios de casos y controles, } \\
\text { y estudios de cohortes con alto } \\
\text { riesgo de sesgo y con riesgo } \\
\text { importante de que la relación } \\
\text { no sea causal. }\end{array}$ & & \\
\hline 3 & $\begin{array}{l}\text { Estudios no analíticos, como } \\
\text { informes de casos y series de } \\
\text { casos. }\end{array}$ & $\begin{array}{l}\text { Evidencia de nivel } 3 \text { ó } 4 \text {, } \\
\text { o evidencia extrapolada desde } \\
\text { estudios clasificados como } 2+\text {. }\end{array}$ & $\mathrm{D}$ \\
\hline 4 & Opinión de expertos. & $\begin{array}{l}\text { Puntos de Buena Praxis: mejor } \\
\text { práctica recomendada basada, } \\
\text { por consenso, en la experiencia } \\
\text { clínica de los miembros de los } \\
\text { grupos que han desarrollado la GPC. }\end{array}$ & $\mathrm{BP}$ \\
\hline
\end{tabular}

En una primera fase dos documentalistas expertos en documentación biomédica, reprodujeron, de forma independiente la estrategia de búsqueda. En una segunda etapa dos revisores del ámbito de la medicina del trabajo verificaron las publicaciones identificadas y realizaron una primera selección de publicaciones de acuerdo a los criterios de selección. En una tercera fase un equipo multidisciplinar de cuatro investigadores (del ámbito de la medicina del trabajo, epidemiología y metodología de investigación) mediante revisión por pares, cribaron los estudios al objeto de consolidar la selección realizada. En caso de discrepancia entre pares se llegó a un acuerdo mediante consenso. 


\section{RESULTADOS}

En total se recuperaron un total de 236 referencias de publicaciones entre 1995 y 2008 cuya distribución por descriptor se refleja en la tabla 4.

Tabla 4: Referencias recuperadas por término "Mesh o descriptors" utilizados.

\begin{tabular}{lc}
$\begin{array}{l}\text { ESTRATEGIA DE BÚSQUEDA } \\
\text { (términos “MeSH o descriptors”) }\end{array}$ & $\begin{array}{c}\text { REFERENCIAS } \\
\text { RECUPERADAS }\end{array}$ \\
\hline Noise AND Cardiovascular diseases & $\mathbf{2 1}$ \\
Occupational noise AND Cardiovascular Diseases ](**) & 20 \\
Occupational noise AND Hypertension] Limits: (*) & 46 \\
Occupational noise AND (Hypertension OR Blood Pressure) AND (1995 (...) OR 2008)) & 2 \\
Occupational noise AND Myocardic infartion & 5 \\
Occupational noise AND (Myocardic infartion OR Coronary Disease) ] (**) & 8 \\
((“Cardiovascular Diseases”[Mesh] OR “Hypertension”[Mesh]) OR “Coronary & 7 \\
Disease”[Mesh]) AND Occupational noise (*) & 236 \\
Noise AND (Coronary heart disease OR Coronary heart diseases OR Coronary disease) & \\
\hline TOTAL & \\
\hline
\end{tabular}

Tras aplicar los filtros descritos anteriormente y los criterios de inclusión y exclusión se seleccionaron un total de veintitrés artículos para revisión a texto completo (tabla 5).

En la producción científica analizada predominaron los estudios de diseño transversal (doce artículos), seguidos de los diseños de cohorte (siete artículos), estudios casos control (dos artículos), recuperándose sólo un meta-análisis y un estudio experimental. 
Tabla 5: Referencias estudiadas sobre Enfermedades Cardiovasculares e Hipertensión por Tipo de estudios y efectos estudiados

\begin{tabular}{|c|c|c|c|c|}
\hline Ref. & $\begin{array}{l}\text { PRIMER } \\
\text { AUTOR }\end{array}$ & AÑ̃ & $\begin{array}{l}\text { TIPO de } \\
\text { DISEÑO }\end{array}$ & EFECTOS ESTUDIADOS \\
\hline (24) & Virkkunen & 2005 & Cohorte & Incremento de enfermedad cardiovascular \\
\hline (25) & Virkkunen & 2006 & $\begin{array}{l}\text { Cohorte } \\
\text { prospectiva }\end{array}$ & Aumento de riesgo de enfermedad coronaria \\
\hline (26) & Virkkunen & 2007 & $\begin{array}{l}\text { Cohorte } \\
\text { prospectiva }\end{array}$ & $\begin{array}{l}\text { Aumento de Tensión arterial sistólica y aumento } \\
\text { de riesgo de enfermedad coronaria }\end{array}$ \\
\hline (27) & Sbihi & 2008 & Cohorte & Hipertensión \\
\hline (28) & Davies & 2005 & $\begin{array}{l}\text { Cohorte } \\
\text { prospectiva }\end{array}$ & Riesgo de mortalidad por IAM \\
\hline (29) & Melamed & 2001 & Cohorte & Hipertensión \\
\hline (30) & Fujino & 2007 & Cohorte & $\begin{array}{l}\text { Enfermedad cerebrovascular (hemorragia } \\
\text { intracraneal e hipertensión) }\end{array}$ \\
\hline (31) & McNamme & 2006 & Casos Control & Mortalidad cardiovascular,riesgo de infarto \\
\hline (32) & Willich & 2006 & Casos Control & Riesgo de mortalidad por IAM \\
\hline (33) & Kristal -Boneh & 1995 & Transversal & $\begin{array}{l}\text { Aumento agudo de tensión arterial y frecuencia } \\
\text { cardiaca }\end{array}$ \\
\hline (34) & Melamed & 1997 & Transversal & $\begin{array}{l}\text { No relación entre ruido y lípidos, excepto hDL } \\
\text { en mujeres }\end{array}$ \\
\hline (35) & Talbott & 1999 & Transversal & Hipertensión arterial \\
\hline (36) & Tomei & 2000 & Transversal & $\begin{array}{l}\text { Alteraciones en EKG y aumento de tensión arterial } \\
\text { sistólica y diastólica }\end{array}$ \\
\hline (37) & Fogari & 2001 & Transversal & Aumento de frecuencia cardiaca y tensión arterial \\
\hline (38) & Prowaska & 2003 & Transversal & Aumento hipertensión (TAS) \\
\hline (39) & Lusk & 2002 & Transversal & Disminución de TAS y TAD con protector auditivo \\
\hline$(40)$ & Lusk & 2004 & Transversal & Aumenta TAS y F cardiaca \\
\hline (41) & Jing Yuan & 2005 & transversal & Isquemia crónica de miocardio, alteraciones EKG \\
\hline$(42)$ & Inoue & 2005 & Transversal & Hipertensión \\
\hline (43) & Chang & 2007 & transversal & $\begin{array}{l}\text { Hipertensión, parámetros vasculares (complianza, } \\
\text { distensibilidad y Resistencia) }\end{array}$ \\
\hline$(45)$ & $\mathrm{Ni}$ & 2007 & transversal & Hipertensión y parámetros vasculares \\
\hline (46) & Van Kempen & 2002 & Metanálisis & Hipertensión \\
\hline (48) & Holand & 1999 & Experimental & Hipertensión \\
\hline
\end{tabular}




\section{Estudios de Cohortes}

Virkunnen $H$ \& col en el año $2005^{24}$ Realizó un estudio de cohorte prospectivo de 18 años de seguimiento, perteneciente al Helsinki Heart Study (HHS), sobre los efectos a corto y largo plazo de la exposición laboral a ruido continuo y de impacto en relación al riesgo de padecer enfermedad coronaria.

La muestra fue de 6.005 varones trabajadores de la industria, el seguimiento se realizó en tres momentos (años 1991-1995-1999). Clasificaron a los trabajadores en trabajadores de "cuello blanco" y trabajadores de "cuello azul" según categorías profesionales del censo finlandés.

Los Eventos Coronarios (CIE 9-10) se determinaron por registros de altas hospitalarias y de morbilidad.

La exposición se evaluó de forma cualitativa mediante la matriz de exposición global laboral finlandesa (FINJEM). La exposición a ruido continuo se clasificó en tres umbrales $<80 \mathrm{dBA}, 80-85 \mathrm{dBA},>85 \mathrm{dbA}$. La exposición a ruido de impacto y ruido combinado se categorizó en expuestos y no expuestos.

Se ajustó el análisis por factores de confusión para enfermedad coronaria.

Entre los resultados encontrados más significativos destaca que entre 1982 y 1999 se produjeron un total de 1.166 eventos coronarios, de los cuales, 164 tuvieron desenlace fatal. A los 9 años de seguimiento se encontró un Riesgo Relativo (RR) de 1,38 (95\% IC 1,04-1,82) y a los 18 años de seguimiento, el RR fue de 1,54 (IC 95\% 1,28-1,86).

Para trabajadores de cuello azul el RR resultó ser significativo a los 13 años de seguimiento siendo de 1,29 (IC del 95\% 1,05-1,57). El ajuste con otros factores de riesgo para ECV no variaron los resultados.

La exposición a ruido de impacto durante un periodo de 18 años se asoció con un aumento del riesgo para ECV, incremento que se mantuvo incluso en la población jubilada ( $\mathrm{p}<<$ significativa).

Virkkunen $H$ Ecol (2006) ${ }^{25}$ en este estudio, también perteneciente al Helsinki Heart Study (HHS), los autores analizaron la interacción entre trabajo a turnos, ruido y carga física sobre la enfermedad coronaria, estimando el efecto conjunto de estos factores. Se realizó un estudio de cohorte prospectivo de 13 años de seguimiento (19871999), cuya cohorte la formaba 1.804 varones, trabajadores de la industria incluidas en el HHS.

La muestra fue dividida en subgrupos, un subgrupo expuesto a ruido continuo, un subgrupo expuesto a ruido combinado (ruido de impacto y continuo) y un subgrupo de trabajadores no expuestos, formado por trabajadores de tareas administrativas.

La exposición a otros factores de riesgo de origen laboral se analizó mediante la matriz de empleo-exposición finlandesa (FINJEM).

Las posibles variables de confusión y sesgos se controlaron en la selección. Los criterios de selección y de exclusión se describen correctamente.

Los riesgos relativos se estimaron usando el modelo de Cox y se ajustaron por posibles factores de confusión para la enfermedad coronaria.

A los cinco años de seguimiento, se encontraron RR superiores a 1 asociados a la exposición a ruido combinado con un RR de 1,28 y para carga física con un RR de 1,18, en ambos casos los intervalos de confianza no eran significativos. Sin embargo, para trabajo a turnos el RR fue de 1,59 con intervalos de confianza significativos (IC 95\%1.10-2.31).

El RR fue más elevado para padecer ECV cuando el trabajo a turnos se combinaba con exposición a ruido. 
En 2007 Virkkunen $H \in \operatorname{col}^{26}$ realizan un estudio similar en la misma cohorte analizando la relación entre exposición a ruido, trabajo a turnos y carga física en relación con la tensión arterial sistólica.

En un estudio de 8 años de seguimiento, analizan, la exposición a ruido, trabajo a turnos y carga física y su relación con el incremento de la tensión arterial sistólica basal (TS) y el desarrollo de enfermedades coronarias. La muestra estudiada fue de 884 trabajadores para el estudio sobre la TS y de 1.288 trabajadores para el estudio de ECV.

De acuerdo a los resultados de este estudio la exposición a ruido no aumenta, por si misma, la TS, pero sí lo hace cuando interviene de forma combinada con el trabajo a turnos y/o la carga física.

Sbihi H. $E$ col $^{27}$ en el año 2007 realizan un estudio de cohorte retrospectivo en 10.872 varones trabajadores de una empresa maderera sobre la incidencia de HTA durante un periodo de 7 años (1991-1998).

La cohorte se constituyó incluyendo trabajadores vivos en la fecha de inicio del periodo de seguimiento. La definición de caso se realizó de acuerdo a las guías de detección y diagnostico de HTA de los servicios de salud de la zona de Columbia Británica, encontrándose un total de 828 casos, que fueron agrupados en las siguientes categorías: fallecidos, presentar al menos un ingreso hospitalario y/o tres visitas al médico por HTA dentro de un periodo de 70 días.

En cuanto a la exposición a ruido, se excluyeron los trabajadores con una exposición a ruido $<$ de $85 \mathrm{dBA}$.

Se analizó la duración y el nivel de exposición en los umbrales de >85 dBA, >90 dBA y >95 dBA. El estudio no valora el uso de EPIs; ni se realiza un ajuste por otros posibles confusores, salvo la edad.

Los RR ajustados por edad fueron de 1,5 (IC 95\% 1,1 -2) en trabajadores expuestos durante más de 29 años a 85 dBA; existiendo una relación dosis-respuesta con tendencia a ser estadísticamente significativa en los tres umbrales de exposición (valores de $\mathrm{p}=0.002$, 0.006 y 0.0036 respectivamente).

Davies $H$. W E col, dos años antes ${ }^{28}$ realizó un estudio de cohorte retrospectivo entre 1950 y 1995, para estudiar la relación entre la exposición laboral a ruido y la mortalidad por infarto agudo de miocardio (IAM). Estudia una población 27.464 trabajadores "de cuello azul" de la misma empresa maderera.

Los criterios de inclusión en la cohorte fueron al menos haber trabajado un año, durante los 45 años de seguimiento. Incluyó además un subgrupo de 8.668 trabajadores que no utilizaban equipos de protección auditiva.

La definición de caso se realizó utilizando la CIE9 y la mortalidad se obtuvo del registro de mortalidad canadiense. La exposición profesional al ruido se evaluó teniendo en cuenta la exposición acumulada (dBA-año, <100dBA ,100-104.9dBA, 105-109.9dBA, 110-114.9,>115dBA), la duración de la exposición en años y "umbrales de ruido (>85$>90->95)$.

Se controló el sesgo de trabajador sano y diferentes factores de confusión, entre los que se incluía el tabaco y otras sustancias cardiotóxicas de origen laboral. Para controlar el error de clasificación, asociado al uso de equipos de protección, se introdujo en el estudio el seguimiento un subgrupo de trabajadores que no usuarios de estos equipos.

Durante los 45 años de seguimiento del estudio, se produjeron 2.510 muertes por ECV y 1.627 en el subgrupo que no utilizaba protección acústica.

El riesgo para de padecer IAM debido a la exposición laboral a ruido elevado fue mayor tanto para la cohorte general como para el grupo sin protectores auditivos en relación a la población no expuesta. 
Se encontró un RR de 1,5 (IC 95\%1,1-2,2) para mortalidad debida a IAM en el grupo de mayor exposición acumulada a ruido ( $>=115 \mathrm{dBA}$ ). El RR fue de 2,0 en el grupo de trabajadores con mayor tiempo de exposición ( $>19$ años) y umbrales superiores a 85 dBA, ambos con intervalos de confianza estadísticamente significativos.

De acuerdo a los resultados de este estudio la exposición mantenida a altos niveles de ruido se asocia a un aumento de riesgo de muerte por IAM.

Melamed S. E $\operatorname{col}^{29}$ realizan, en 2001 un estudio de cohorte, perteneciente al estudio CORDIS con un periodo medio de seguimiento entre 2 y 4 años, en 807 trabajadores pertenecientes a 161 actividades industriales.

Analiza la relación entre exposición laboral a ruido, complejidad de la tarea y cambios en la tensión arterial (TA) y la satisfacción laboral.

La evaluación del nivel de exposición a ruido se realizó, mediante sonómetro, al inicio y finalización del periodo se seguimiento.

El estudio recogió otras variables de exposición laboral, estabilidad de la exposición de ruido en el tiempo, temperatura ambiente, complejidad del trabajo y satisfacción en el trabajo. Se controlaron variables como: edad, sexo, Historia Familiar de HTA, uso de EPIs, tiempo de permanencia en el puesto de trabajo y categoría laboral.

El estudio de Melamed pone de manifiesto que la TA aumenta más del doble entre los trabajadores expuestos a alto nivel de ruido, incremento que es superior si la exposición a ruido se combina con la realización de tareas complejas.

Fujino, Y. $2007^{30}$ Estudió una cohorte de 14.568 trabajadores varones perteneciente a la Cohorte japonesa del estudio JACC, compuesta por 110.792 personas.

En este estudio se analizó mediante un cuestionario auto administrado la asociación entre exposición percibida a ruido en el trabajo y el riesgo de sufrir una ECV, para lo que se tomó la CIE10 como referencia en la definición de caso (enfermedad cerebrovascular, hemorragia subaracnoidea, hemorragia intra cerebral o infarto cerebral).

En el estudio se controlaron posibles factores de confusión como: tabaco, alcohol, HTA y diabetes, nivel de educación, stress mental percibido, horas que caminan, horas de ejercicio, tipo de trabajo, turno de trabajo dominante.

Los resultados del estudio, mediante el análisis de los datos por un modelo proporcional de Cox, pusieron de manifiesto que la exposición a ruido percibida en el trabajo aumenta el riesgo de hemorragia cerebral (RRs 2.38, IC 95\% 1.20-4.71, p=0,003), sin embargo no se evidenció un incremento de riesgo ni para la enfermedad cerebrovascular, hemorragia subaracnoidea, o infarto cerebral.

En la tabla 6 se resumen las principales características de los estudios de cohorte analizados para los efectos Cardiovasculares e HTA. 


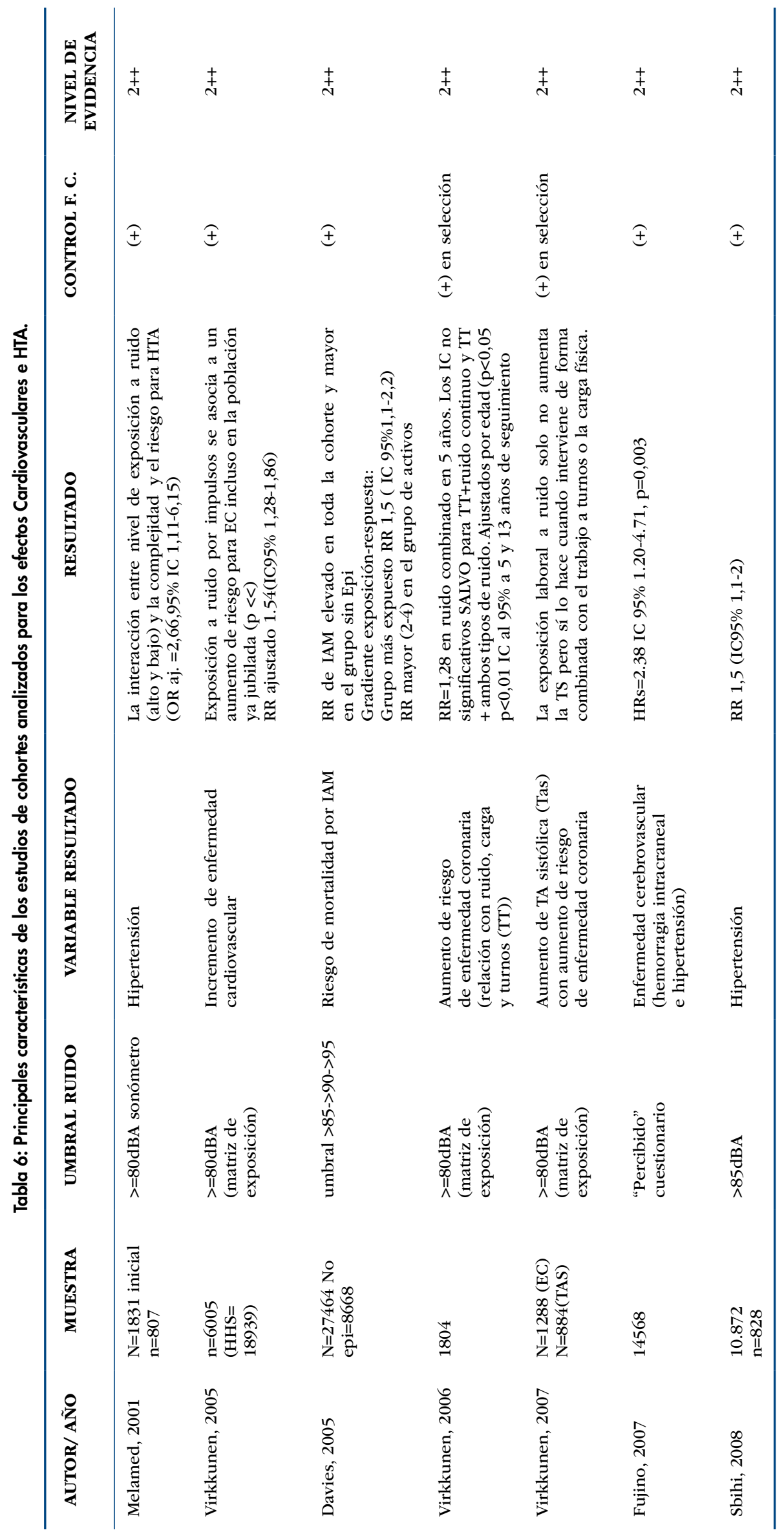




\section{Estudios Casos-Control}

Mc Namee $R$ \& col. $2006^{31}$ Estudió el riesgo de mortalidad por enfermedad isquémica cardiaca (IAM) y la exposición profesional a ruido mediante un diseño casos control en dos sedes diferentes (sede A y sede B) de una central nuclear inglesa, con 1.101 pares estudiados (1:1). Estudio anidado en una cohorte de la misma empresa con seguimiento de 1950 a 1998.

Se definió como caso a los trabajadores de $<=75$ años, muertos por IAM. Para cada caso se eligió un control vivo de la cohorte, apareados por edad, lugar y tiempo de trabajo.

La exposición a ruido se evaluó de forma retrospectiva mediante el análisis de registros por 3 técnicos expertos, de forma independiente, estimándose los siguientes índices de exposición: TT85 (total de años con exposición estimada de ruido), M85 (media de intensidad de la exposición de ruido) y NIL85 (exposición acumulada).

Se controlaron posibles factores de confusión o modificadores del efecto como: uso protectores auditivos. TA, peso, talla, índice de masa corporal (IMC) y tabaco, en el momento de ingreso en la empresa.

Mac Namee no encontró, en la sede A, una asociación significativa entre exposición profesional a ruido y enfermedad isquémica. Sin embargo en la sede B la OR fue de 1,45 (IC de 95\%1,02-2,06) en el nivel "medio" de exposición a ruido, significación que no se mantuvo cuando se ajustó por duración de empleo (al menos 5 años de permanencia en el puesto de trabajo): $\mathrm{OR}=1,33(0,88-2,01)$.

Willich S.N E col $2006^{32}$ Realizaron un estudio de Casos-control, con 4.115 pacientes hospitalarios diagnosticados de IAM entre 1998 y 2001, en 32 hospitales de Berlín. Los controles son elegidos del mismo hospital, sexo y edad que los casos.

Se estudió la exposición a ruido ambiental y ocupacional a través de mapas de ruido, mediante un cuestionario de sobre malestar por exposición a ruido.

El ruido ambiental se asoció a un incremento del riesgo de infarto en hombres y mujeres (OR 1,45 IC 95\% 1,02-2.09p=0,040 y OR 3,36, 1,4-8,06 p 0,007). La exposición profesional a ruido sólo se asoció a un aumento de riesgo de infarto en hombres.

La inferencia a población general es la principal limitación a considerar en este estudio dado que sus resultados serían sólo representativos de pacientes con IAM no fatal.

En la tabla 7 se resumen las principales características de los estudios casos control analizados para los efectos Cardiovasculares e HTA.

Tabla 7: Principales características de los estudios casos control analizados para los efectos Cardiovasculares e HTA.

\begin{tabular}{|c|c|c|c|c|c|c|}
\hline AUTOR/ AÑ̃ & MUESTRA & $\begin{array}{c}\text { UMBRAL } \\
\text { RUIDO }\end{array}$ & $\begin{array}{c}\text { VARIABLE } \\
\text { RESULTADO }\end{array}$ & RESULTADO & $\begin{array}{l}\text { CONTROL } \\
\text { F. C }\end{array}$ & $\begin{array}{c}\text { NIVEL } \\
\text { EVIDENCIA }\end{array}$ \\
\hline $\begin{array}{l}\text { Willich, } \\
2005\end{array}$ & $\mathrm{n}=4115$ & $\begin{array}{l}\text { mapas de } \\
\text { ruido ( } 1-5, \\
10 \text { años) }\end{array}$ & $\begin{array}{l}\text { Riesgo de } \\
\text { mortalidad } \\
\text { por IAM }\end{array}$ & $\begin{array}{l}\text { Ruido ambiental } \\
\text { asociado a } \\
\text { incremento riesgo } \\
\text { infarto en hombres } \\
\text { y mujeres y ruido } \\
\text { ocupacional sólo } \\
\text { en hombres. }\end{array}$ & $(+)$ & $2++$ \\
\hline $\begin{array}{l}\text { Mc Namme, } \\
2006\end{array}$ & $\begin{array}{l}1101 \\
\text { (Sedes A } \\
\text { y B) }\end{array}$ & $<=85 \mathrm{dBA}$ & $\begin{array}{l}\text { Mortalidad } \\
\text { cardiovascular, } \\
\text { riesgo de infarto } \\
\text { de miocardio }\end{array}$ & $\begin{array}{l}\text { Sitio B: OR } 1.45 \\
(1.02-2.06) \text { en } \\
\text { exposición media } \\
\text { pero no asociación } \\
\text { si se ajusta por } \\
\text { duración de empleo } \\
1.33(0.88-2.01) \text {. }\end{array}$ & $\begin{array}{c}\text { (+) en } \\
\text { selección }\end{array}$ & $2++$ \\
\hline
\end{tabular}




\section{ESTUDIOS TRANSVERSALES}

Kristal-Bonet $\&$ Melamed $\&$ col $1995^{33}$ estudiaron los efectos agudos y crónicos de la exposición a ruido industrial sobre la frecuencia cardiaca y la tensión arterial en reposo. Estudio transversal procedente también de la Cohorte CORDIS, que reúne una muestra de 3.105 trabajadores de ambos sexos, de 21 empresas del sector industria con edades comprendidas entre 20-65 años.

Se realizó una entrevista personal para la selección de sujetos. La exposición profesional a ruido se midió mediante sonómetro, evaluándose la exposición a ruido continuo y de impacto.

Los criterios de exclusión se encuentran bien definidos en el estudio y se controlaron posibles factores de confusión mediante análisis multivariante.

Como resultados, mostraron una asociación positiva entre la intensidad de la exposición a ruido y trabajo en turno de mañana con la frecuencia cardiaca en reposo, tanto en mujeres $(\mathrm{p}<0,036)$ como en varones $(\mathrm{p}=0,046)$. No se encontró asociación con la tensión arterial.

Este mismo grupo realizó en 2007 otro estudio transversal en la misma cohorte sobre exposición laboral a ruido, y niveles de lípidos en trabajadores expuestos. No lograron evidenciar una asociación entre la exposición profesional a ruido e incremento de lípidos, excepto para el HDL en mujeres ${ }^{34}$.

Talbott E.E col $1999^{35}$ realizaron un estudio en 500 varones entre 40 y 63 años, que habían permanecido durante 15 años o más, trabajando en dos sedes de la misma empresa. Los trabajadores de la sede 1 se encontraban expuestos a niveles de ruido iguales o superiores a $89 \mathrm{dBA}$, mientras que los niveles de exposición a ruido de los trabajadores de la sede 2 eran inferiores a $83 \mathrm{dBA}$.

Se midió la exposición laboral a ruido y la TA en los trabajadores de ambas sedes, así como otras características clínicas, físicas y de hábitos, relacionadas con el riesgo cardiovascular y la exposición extra laboral a ruido. Se excluyó a los trabajadores que se encontraban en tratamiento con antihipertensivos.

El control de variables de confusión y control de sesgos no queda bien descrito.

Las mediciones de TS realizadas en los trabajadores de ambas sedes, fueron de $123,3 \mathrm{mmHg}$ en la sede 1 (niveles de exposición a ruido iguales o superiores a 89dBA) y $12,8 \mathrm{mmhg}$ en la sede 2 (niveles de exposición a ruido iguales o inferiores 83dBA) $(\mathrm{p}=0,06)$. La tensión arterial diastólica (TD) en los trabajadores de la sede 1 fue de $80,3 \mathrm{mmhg}$ y de $77,8 \mathrm{mmHg}(\mathrm{p}=0,014)$, en la sede 2 .

Tomei F., Fantini S. E col. (2000) ${ }^{36}$ realizan un estudio en una industria del metal, sobre la relación entre la exposición laboral a ruido su intensidad y tipo y la prevalencia de ECV. En este estudio se analizó también la relación entre daño auditivo e HTA.

Se estudiaron tres grupos de trabajadores con diferente nivel de exposición: Un primer grupo integrado por 52 trabajadores que presentaban diferentes déficits de auditivos y una exposición a ruido superior a $90 \mathrm{dBA}$.

Un segundo grupo integrado por 65 trabajadores sin déficit auditivo y exposición a ruido inferior a $60 \mathrm{dBA}$, y un tercer grupo de 64 trabajadores administrativos sin exposición a ruido ni déficit auditivo.

La exposición a ruido se midió mediante sonómetro y se realizó una audiometría con 16 horas de reposo acústico.

Dentro del grupo de trabajadores expuestos a más de $90 \mathrm{dBA}$, la TD media y la prevalencia de HTA fue significativamente más elevada que en los grupos de trabajadores con una exposición inferior $90 \mathrm{dBA} /$ día.

Fogari R. E col $2001^{37}$ Estudian a 476 trabajadores normotensos de una empresa metalúrgica, con edades comprendidas entre los 20 y los 50 años, al objeto de observar 
si la exposición profesional a ruido se asocia a un aumento de la tensión arterial (TA) y si existen diferencias entre expuestos y no expuestos, para la tensión arterial sistólica (TS), tensión arterial diastólica (TD) y frecuencia cardiaca (FC).

El grupo de expuestos ( $>85 \mathrm{dBA}$ ) estaba compuesto por 238 trabajadores y el grupo de no expuestos lo formaban un total de 238 trabajadores (<80dBA).

La evaluación de la exposición a ruido se realizó mediante dosímetros y MAPA de 24 horas. Para la TA se realizaron dos mediciones, una medición durante en días laborales y en otra en días de descanso, con un intervalo máximo entre ambas mediciones de 14 días.

En el estudio quedaba bien definido tanto el control de sesgos como los criterios de exclusión e inclusión.

En los trabajadores menores de 50 años, la exposición profesional a ruido se asoció con un aumento no mantenido de la TA.

No se encontraron diferencias significativas entre expuestos y no expuestos ni paran la TS $(130,2$ vs 129,1 NS), ni TD $(81,9 / 81,4$ NS), ni para la FC $(76,1+8$ vs $17,3+8$ lpm NS) entre expuestos y no expuestos

Powazka, E. E col. $2003^{38}$ estudian los cambios de la TA y la exposición profesional al ruido en 178 trabajadores de una industria siderúrgica, los trabajadores estudiados tenían una edad superior a 35 años, sin antecedentes de HTA, con una antigüedad en la empresa de más de 3 años. Consideran dos niveles de exposición, exposición superior $85 \mathrm{dBA}$ y exposición inferior a $85 \mathrm{dBA}$.

Para evaluar el estado de salud se utilizó un cuestionario de salud y se realizó una audiometría, medición de la tensión arterial entre otras.

Metodológicamente utilizaron un análisis de comparación de medias, correlación y regresión con variables dependientes (presión sistólica y diastólica), controlando posibles factores de confusión como: tabaco, dieta y otros factores asociados al riesgo de hipertensión.

Los resultados pusieron de manifiesto un incremento significativo de la TS en trabajadores expuestos a altos niveles de ruido, pero no de la TD.

Lusk $2002^{39}$ estudian a los efectos de la exposición profesional a ruido, la utilización de protectores auditivos (EPI) y su relación con el aumento de la TA y cambios en la FC en 374 trabajadores de una fábrica de automóviles.

La evaluación de la exposición a ruido se realizó de forma retrospectiva tomando los valores de exposición a partir de los datos de las historias médico-laborales durante los 5 años previos al estudio. Controlaron potenciales factores de confusión.

Los resultados mostraron que los trabajadores expuestos a ruido y usuarios de EPI presentaban valores de TS y TD significativamente más bajos que los valores presentados por los trabajadores expuestos no usuarios de este tipo de protección. La frecuencia cardiaca no se modificó con el uso de EPIs.

Lusk 2004 este mismo grupo realizó dos años más tarde un estudio transversal de similares características, en una muestra de 46 trabajadores para estudiar el efecto de la exposición aguda a ruido laboral sobre la tensión arterial y la frecuencia cardiaca. Para medición de la exposición utilizan MAPA y dosímetros personales.

Los autores concluyen que puede existir asociación positiva entre la exposición profesional a ruido y la TS, TD y FC ${ }^{40}$.

Jing Yuan \& col, $2005^{41}$ Analizan la relación entre la exposición profesional a ruido y el aumento en plasma de anticuerpos anti Hsp70 y anti Hsp60 y anormalidades en Electrocardiograma (ECG).

El estudio se realizó en 396 trabajadores de la industria automovilística china de ambos sexos y en 55 puestos de trabajo. El grupo de trabajadores expuestos lo formaban 
un total de 215 trabajadores con una exposición al menos de 1 año, el resto, 181 trabajadores, no se encontraban expuestos a ruido.

La evaluación de la exposición se realizó mediante sonómetro con tres mediciones al día, durante dos días consecutivos y dos veces al año.

Los autores concluyen que el aumento del nivel de Hsp70n (estimulado por ruido) se encuentra asociado a un mayor riesgo de alteraciones en ECG como isquemia crónica miocárdica $(\mathrm{p}<0,05)$. El aumento de Hsp60 se asoció a anormalidades del ECG tipo arritmia sinusal, isquemia crónica cardiaca, y ritmo ectópico. Se encontraron altos niveles de hsp70-60 asociados a un incremento de anormalidades en el ECG, ajustado por nivel de exposición a ruido (anti Hsp70 OR = 1,73,1,04-2,86 y anti Hsp $60 \mathrm{OR}=1,36,1,07-1,72$ )

Masaiwa Inoue, $2005^{42}$ realizaron un estudio en 415 trabajadores de una empresa de manufactura de papel sobre la relación entre la exposición laboral a ruido y el aumento de la TA. 242 trabajadores se encontraban expuestos a ruido (92dBA) y usaban protectores auditivos. El grupo control lo componían un total de 173 trabajadores de la misma empresa que trabajaban en el departamento de química, con una exposición a ruido (75dBA) y sin uso de protectores auditivos.

Los criterios de inclusión, de medición y de recogida de datos se encuentran bien definidos. El estudio se ajusta por factores de confusión como ingesta de alcohol, tabaco, edad, historia familiar, sedentarismo y dieta.

Los autores encuentran, en el grupo de trabajadores expuestos, una prevalencia de hipertensión del 6,9\% (OR = 0,48, IC95\% 0,28-0,81), y una asociación inversa y significativa entre HTA y exposición laboral a ruido.

Concluyen afirmando que los trabajadores usuarios de EPI presentaron valores de TA más bajos.

Ta-Yuan Chang. E col (2007) ${ }^{43}$ realizaron un estudio transversal en fábricas de automóviles en Taiwán con un tamaño muestral de 20 trabajadores.

Se analizaron parámetros vasculares de arteria braquial y valores de complianza, distensibilidad y resistencia en relación a diferentes niveles de exposición a ruido.

El estudio concluyó que a niveles de exposición de 85dBA aumenta significativamente la resistencia sistémica vascular (SVR) y disminuye la complianza y distensibilidad de la arteria braquial (BAC, BAD) y la complianza sistémica vascular (SVC). Los autores sostienen que la exposición profesional a ruido podría favorecer efectos vasculares e HTA.

Este mismo autor realizó en 2003, un estudio descriptivo de características y muestra similar, en la misma empresa. Se consideraron niveles de exposición >85 dBA (hasta 120dBA) durante 8 horas y sin utilización de equipos de protección individual.

Los autores observaron una relación entre exposición a ruido y tensión arterial.

Las variables de confusión no estaban suficientemente controladas en el estudio ${ }^{44}$.

Ni Chun-hui. E col, $2007^{45}$ estudian mediante un diseño transversal en 618 trabajadoras de la industria textil, sanas de edades medias de 35,8 $\pm 6,1$ y expuestas profesionalmente a ruido durante 10,6 \pm 7,6 años, con un umbral de exposición entre 80,1 y 113 dBA.

El estudio divide a las trabajadoras en un grupo con pérdida auditiva audiométrica (151) y otro de audición normal (467).

La pérdida auditiva se valoró mediante audiometría, midiendo también la tensión arterial sistólica y diastólica (TS, TD) y la complianza arterial (CA).

Los resultados del estudio, reflejaron datos de perdida auditiva en frecuencias agudas $(24,4 \%)$, déficit auditivos en frecuencias conversacionales $(0,81 \%)$ e incidencia de hipertensión (7,93\%). La TS y TD en el grupo con hipoacusia fue mayor que en el grupo control $(\mathrm{p}<=, 05)$ y la CA también fue menor en el grupo control $(\mathrm{p}<0,05)$.

En la tabla 8 se resumen las principales características de los estudios transversales analizados para los efectos cardiovasculares y la hipertensión. 


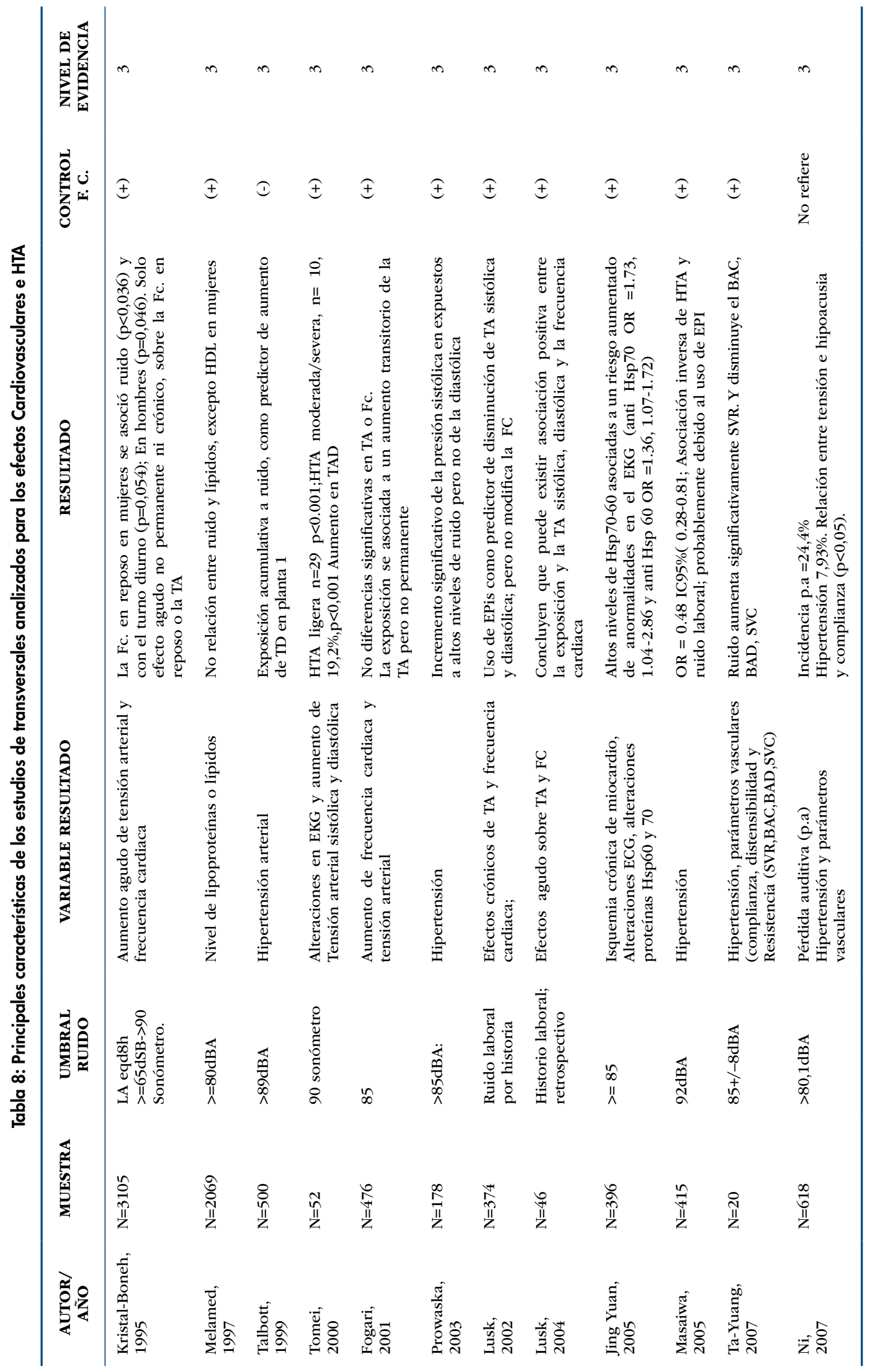




\section{Meta análisis}

Van Kempen E. E col $2002^{46}$. Realizan un meta-análisis para evaluar tanto la relación entre la exposición ambiental y laboral a ruido con la TA y/o la enfermedad isquémica cardiaca, definida según CIE9 códigos 410-414.

Analizaron cuarenta y tres estudios epidemiológicos publicados entre 1970-1999 de los cuales veintitrés son transversales, cuatro de cohortes y uno es una serie de casos.

La estrategia de búsqueda y los criterios de inclusión y exclusión están descritos de forma amplia y detallada.

Analizaron las variables de TA, uso de fármacos antihipertensivos, seguimientos por médico general o especialista, uso de fármacos para ECV, angina de pecho, IAM y prevalencia de enfermedad isquémica.

Los resultados de este meta-análisis mostraron una asociación estadísticamente significativa entre exposición profesional a ruido y de la exposición producido por el tráfico con la HTA pero la evidencia es limitada para otros riesgos cardiovasculares o de salud.

Por cada aumento de $5 \mathrm{dBA}$ de ruido en el ambiente laboral el RR $(5 \mathrm{dBA})=1,14$ (IC 95\%1,01-1,29), ajustados por edad, sexo y tipo de trabajo. También se observó un aumento de TS en relación a exposición profesional a ruido de $0,5(0,01-1) \mathrm{mmHg}$. $/ 5 \mathrm{dBA}$

Las conclusiones de esta publicación son coincidentes con las de otros meta-análisis, donde encuentran un aumento de la $\mathrm{OR}$ en función del aumento de ruido por encima de 20 unidades Costeen (equivalente a LAeq7-19 hr 55dbA) ${ }^{47}$.

En la tabla 9 se resumen las principales características del meta-analisis analizado para los efectos cardiovasculares y la hipertensión

Tabla 9: Principales características del meta-análisis analizados para los efectos Cardiovasculares e HTA

\begin{tabular}{llcllcc}
\hline AUTOR/ AÑo & MUESTRA & $\begin{array}{c}\text { UMBRAL } \\
\text { RUIDO }\end{array}$ & $\begin{array}{c}\text { VARIABLE } \\
\text { RESULTADO }\end{array}$ & RESULTADO & $\begin{array}{c}\text { CONTROL } \\
\text { F. C }\end{array}$ & $\begin{array}{c}\text { NIVEL } \\
\text { EVIDENCIA }\end{array}$ \\
\hline $\begin{array}{l}\text { Van Kempen, } \\
2002\end{array}$ & 43 & $\begin{array}{l}\text { Leqd8h:55 } \\
-116 \mathrm{dBA}\end{array}$ & Hipertensión & $\begin{array}{l}\text { RR para ruido } \\
\text { ocupacional: } 1.14 \\
\text { (IC 95\%1.01-1,29) }\end{array}$ & $(+)$ & $1++$ \\
\hline
\end{tabular}

\section{Experimentales}

Holand $S \&$ col $1999^{48}$ realizan un estudio con 25 voluntarios de ambos sexos, donde describen el efecto de un estímulo auditivo agudo sobre la TA y la FC. De ellos 9 eran hipertensos no tratados. Clasificaron la HTA de acuerdo a la Organización Mundial de la Salud (OMS) de tensión arterial: óptima, normal, leve y moderada.

Previo a la exposición se realizó un examen físico cuyo resultado determinó que ninguno padecía hipoacusia y tampoco tomaba fármacos para el tratamiento de problemas relacionados con la TA.

Controlan la acomodación a ruido con un ruido de 55 dBA a través de audífonos, para posteriormente provocar dos estímulos de $110 \mathrm{dbA}, 1-20 \mathrm{HZ}$, durante 0,15 segundos.

Entre los principales resultados se registró un aumento medio en la TS de 18,72 mmHg media a los 5,1 segundos tras el estímulo de ruido, un aumento medio de FC de 10,8+/-1,1 lpm a los 3,4 segundos ( $\mathrm{p}<0,01)$; con el segundo estímulo el aumento fue de menor intensidad pero significativo $(\mathrm{p}<0,01)$. Lo que evidenció un aumento precoz de la TA y FC dentro de los primeros 10 segundos de estímulo a frecuencias agudas probablemente debido a una activación simpática. 
En la tabla 10 se resumen las principales características del estudio experimental analizado para los efectos cardiovasculares y la hipertensión.

Tabla 10: Principales características del estudio experimental analizados para los efectos Cardiovasculares e HTA

\begin{tabular}{|c|c|c|c|c|c|c|}
\hline AUTOR/ AÑO & MUESTRA & $\begin{array}{l}\text { UMBRAL } \\
\text { RUIDO }\end{array}$ & $\begin{array}{c}\text { VARIABLE } \\
\text { RESULTADO }\end{array}$ & RESULTADO & $\begin{array}{l}\text { CONTROL } \\
\text { F. C }\end{array}$ & $\begin{array}{c}\text { NIVEL } \\
\text { EVIDENCIA }\end{array}$ \\
\hline $\begin{array}{l}\text { Holand, } \\
1999\end{array}$ & $\mathrm{~N}=25$ & $\begin{array}{l}110 \mathrm{dBA} \\
1-20 \mathrm{KHz}\end{array}$ & Hipertensión & $\begin{array}{l}\text { Aumento } \\
\text { significativo } \\
(\mathrm{p}<0,01) \text { de TAS } \\
\text { y FC precozmente } \\
(0-10 \mathrm{~s}) \text { aumento } \\
\text { medio de TA de } \\
18.7 \mathrm{~mm} \mathrm{Hg}\end{array}$ & $(+)$ & $1+$ \\
\hline
\end{tabular}

\section{CONCLUSIONES}

En total hemos analizado 23 estudios sobre exposición profesional a ruido y efectos cardiovasculares: 1 meta-análisis, 1 estudio experimental, 7 cohortes, 2 casos controles y 12 estudios transversales.

Los efectos cardiovasculares estudiados en relación con la exposición laboral a ruido incluyeron: hipertensión, mortalidad por infarto agudo de miocardio, patologías relacionadas con alteraciones coronarias, enfermedad isquémica cardiaca, alteración de lípidos, alteración de parámetros vasculares arteriales, cambios en la frecuencia cardiaca, cambios en el electrocardiograma y proteínas en plasma, así como probabilidad de sufrir una enfermedad cerebrovascular.

En relación al nivel de evidencia de la asociación entre exposición profesional a ruido y efectos cardiovasculares, encontramos:

\section{Exposición profesional a ruido y sus posibles efectos sobre la tensión arterial.}

Esta relación es la más documentada, tanto por número de publicaciones como por ser el efecto sobre el que existe un nivel de evidencia más fuerte.

La exposición laboral a ruido y el incremento de la tensión arterial tienen un alto nivel de evidencia, basado en cuatro estudios de cohorte $(2++)$, un meta-análisis (1+) y un estudio experimental (1++).

Existe evidencia (2++) en la asociación entre exposición a ruido durante la vida laboral y el incremento en la incidencia de HTA. Existe también evidencia en el incremento de riesgo para HTA y ECV cuando se produce una exposición combinada a ruido junto con carga física, trabajo a turnos y complejidad de la tarea.

La asociación entre enfermedad cardiovascular y exposición profesional a ruido se encuentra también en ocho estudios transversales, en estos estudios se documentan distintos efectos, entre estos: el incremento súbito de la TA tras la exposición a ruido, afectación aislada de la TD o TS, incremento transitorio de la TA, incremento de la FC y asociación con la pérdida auditiva. En cualquier caso la debilidad del diseño transversal, desde el punto de vista de la evidencia (nivel 3 de evidencia) hace que sus resultados deban ser interpretados como no concluyentes.

La efectividad de la protección auditiva en la reducción de la prevalencia de estos efectos en población laboralmente expuesta a ruido dispone también de evidencia, encontrándose relaciones inversas en la asociación efecto/exposición y disminuyendo las cifras de valores de tensión arterial, si bien este efecto se ha analizado con diseños transversales. 
2. Exposición profesional a ruido y sus efectos sobre la enfermedad isquémica cardiaca, enfermedad coronaria o enfermedad cardiovascular

El nivel de evidencia entre exposición profesional a ruido y enfermedad isquémica, coronario o cardiovascular es de $2++$, basado en dos estudios de cohortes y un estudio casoscontrol, los resultados de estos tres estudios son coincidentes en la asociación entre exposición a ruido y enfermedad cardiovascular así como en el incremento del riesgo cuando esta exposición se combina con turnicidad, edad, complejidad de la tarea y ruido de impacto.

\section{Exposición profesional a ruido y sus efectos sobre el infarto agudo de miocardio}

La relación entre exposición profesional a ruido y padecer infarto de miocardio dispone de un nivel de evidencia $2++$, basada en un estudio de cohorte y un estudio casos- control. En el estudio de cohorte la mortalidad por infarto agudo se asocia con un mayor tiempo de exposición a altos niveles de ruido, de forma mantenida y en trabajadores con mayor antigüedad.

\section{Exposición profesional a ruido y sus efectos sobre la frecuencia cardiaca}

La influencia de la exposición profesional a ruido y el incremento de la frecuencia cardiaca disponen de evidencia científica con nivel $1+$ y $2++$, evidencia que procede de un estudio experimental y un estudio de cohorte. En diseños transversales la evidencia es contradictoria, dos estudios encuentran asociación y dos rechazan su existencia.

5. Exposición profesional a ruido y sus efectos sobre las alteraciones bioquímicas y de lípidos

Con respecto a esta relación la evidencia procede de dos estudios transversales, es por lo tanto débil ${ }^{3}$, si bien las conclusiones de ambos son coincidentes en poner de manifiesto la relación entre exposición laboral a ruido y presencia de alteraciones bioquímicas y de lípidos.

6. Exposición profesional a ruido y sus posibles efectos sobre alteraciones en los parámetros vasculares

Las evidencias existentes con respecto a la influencia de la exposición profesional a ruido y alteraciones en parámetros vasculares se basan en dos estudios transversales, por lo tanto con niveles bajos de evidencia ${ }^{3}$, cuyos resultados son coincidentes, concluyendo en la existencia de una asociación entre la exposición a ruido con la distensibilidad y complianza arterial.

\section{Umbrales de exposición a ruido}

Los niveles de exposición profesional a ruido presentan gran variabilidad en los diferentes estudios revisados, que oscilaron desde los 80dBA hasta los $113 \mathrm{dBA}$.

Los niveles de exposición a ruido en relación a la aparición de infarto agudo de miocardio, se sitúan en algunos estudios entre $>=85$ y $>100 \mathrm{dBA}$.

Los niveles de exposición a ruido en relación a cambios en la frecuencia cardiaca se sitúan, de acuerdo a los estudios revisados entre $>=65$ y >90dBA.

Los niveles de exposición a ruido en relación a las alteraciones de parámetros vasculares y alteraciones de proteínas que puedan modificar el ECG, se sitúan por encima de $\operatorname{los}>=85 \mathrm{dbA}$.

El umbral de exposición a ruido para el riesgo de padecer enfermedad coronaria o enfermedad isquémica cardiaca se sitúa entre 80 y $85 \mathrm{dbA}$.

\section{DISCUSIÓN}

Hasta la fecha en que realizamos nuestra revisión, Smith and Broadbent ${ }^{6}$, encontraron una evidencia considerable acerca de la influencia de exposición a ruido en el puesto de trabajo sobre funciones cardiovasculares y niveles hormonales, como las catecolaminas. 
Posteriormente, en la revisión llevada a cabo por Butler y cols. ${ }^{15}$ sobre estudios epidemiológicos más recientes (1988-1994), concluyeron que la exposición a ruido discontinuo o de impacto tiene efectos cardiovasculares y bioquímicos, pero no pudieron demostrar suficientemente la evidencia de estas alteraciones en el caso de la exposición a ruido continuo.

En nuestra revisión hemos encontrado algunos estudios donde se concluye que la exposición a ruido discontinuo se asocia con un mayor riego de padecer enfermedad coronaria y con aumentos transitorios (no mantenidos), de la tensión arterial.

Butler y cols. revisaron múltiples publicaciones que ponían de manifiesto la asociación entre exposición profesional a ruido y alteración de parámetros fisiológicos y bioquímicos, que desencadenaban alteraciones en la tensión arterial ${ }^{50-56}$.

Estos autores refieren un estudio de revisión en la que un 48\% de los estudios analizados referían asociación positiva entre ruido y enfermedad cardiovascular ${ }^{57}$ siendo la hipertensión arterial el factor que con mayor frecuencia se encontraba alterado.

En nuestra actual revisión, esta tendencia se mantiene y se manifiesta en estudios en los que se encuentra un nivel de evidencia elevado $(1++, 1+, 2++)$ y en varios estudios de corte transversal con nivel de evidencia 3, lo que demuestra la asociación entre ruido ocupacional y cambios en la tensión arterial.

El resto de las publicaciones revisadas por Butler y cols, inciden más sobre el estudio de otros factores de riesgo de tipo ambiental y laboral, que pueden influir sobre la aparición de ECV, tales como el sedentarismo o el trabajo a turnos. Nuestros resultados concuerdan con los de Butler, encontramos que estos factores de riesgo se encuentran fuertemente asociados a la posibilidad de padecer ECV como lo demuestran estudios con niveles de evidencia $2++{ }^{26}$.

En el caso de patologías cardiovasculares como el infarto agudo de miocardio en cuanto a su asociación con la exposición profesional a ruido un estudio casos control de 1992 no encuentra una asociación concluyente ${ }^{58}$.

En nuestra revisión, esta asociación es uno de los hallazgos más relevantes dado que hay estudios de evidencia 2++, que han demostrado una fuerte asociación entre exposición profesional a ruido y posibilidad de sufrir IAM. Esta asociación se dio en hombres y no en mujeres.

En relación a la exposición profesional a ruido y su asociación con otras enfermedades como la enfermedad cerebrovascular o el ICTUS, en revisiones previas no se encontraban estudios. Nuestra revisión ha encontrado un estudio de nivel de evidencia $2++$ en el que se concluye que la percepción de exposición a ruido en el medio laboral aumenta el riesgo de hemorragia cerebral, hemorragia subaracnoidea o infarto cerebral, aunque no de enfermedad cerebrovascular ${ }^{30}$.

La asociación entre ruido e hipertensión arterial parece más probada que su asociación con otras enfermedades cardiovasculares como la enfermedad cerebrovascular o el ICTUS. Sería conveniente realizar más estudios en esta dirección, debido a la importancia que a nivel sanitario representa esta patología por su relación con la pérdida de calidad de vida y con la mortalidad.

Otros estudios encontrados analizan la influencia de la exposición a ruido en su relación a la personalidad y cambios en la tensión arterial o efectos cardiovasculares que representa respuestas mediadas por cambios psicológicos más que por patrones fisiológicos ${ }^{51}$. En la esfera psicofisiológica, en nuestra revisión sólo hemos encontrado un estudio de cohortes, también realizado por el mismo grupo que relaciona la exposición profesional a ruido, las tareas complejas y la satisfacción laboral con un aumento de tensión arterial pero sin analizar parámetros psicológicos como el de la personalidad u otros.

Por último, en la mayoría de los estudios de la revisión de Butler y cols. se observa una respuesta bioquímica y fisiológica inmediata a nivel cardiovascular y se encuentran cambios bioquímicos a largo plazo inducidos por la exposición prolongada a ruido. 
Nosotros también hemos encontrado estudios que describen este tipo de reacciones tras la exposición a ruido, aunque no se relacionan con cambios permanentes ${ }^{33 ; 37}$, estos resultados se asientan en estudios con nivel de evidencia 3.

Podemos concluir diciendo que el conocimiento sobre efectos extra-auditivos a nivel cardiovascular, tras la exposición profesional al ruido se basa en diseños que permiten generar niveles altos de evidencia: meta-análisis (1 estudio), diseños experimentales (1 estudio) y analíticos (9 estudios), además de los estudios transversales.

Como avances a destacar, respecto a las anteriores revisiones encontramos que:

- Existen más estudios y de mayor complejidad metodológica en el periodo 1995- 2008, frente a los periodos analizados en anteriores revisiones.

- La mayoría de los estudios describen correctamente los métodos de selección de los sujetos estudiados, indicando las pérdidas de sujetos y sus causas.

- En general, los estudios intentan resolver las limitaciones metodológicas como las mediciones ambientales incorrectas, retrospectivas o dicotómicas del ruido, mediante mediciones ambientales objetivas con sonómetros, de forma normalizada e incluso utilizando dosímetros personales.

- También podemos observar que el control de los sesgos se establece desde la etapa de diseño y los análisis se ajustan, en la mayoría de estudios revisados, por potenciales factores de confusión.

- Por último, se observa una mejor definición de las variables efecto, entre otros aspectos que se encuentran mejorados en los estudios recientes.

Estos resultados ponen de manifiesto que el control del ruido en entornos laborales es un factor de mejora de salud y prevención del riesgo cardio-vascular. Los efectos como HTA, riesgo coronario y otras enfermedades cardiovasculares deben considerarse en los protocolos de vigilancia médica específica de los trabajadores expuestos a ruido.

\section{BIBLIOGRAFÍA}

1. Fundación Europea para la Mejora de las Condiciones de Vida y de Trabajo. Tercera encuesta europea sobre las condiciones de trabajo. 2000.

2. Parent-Thirion A, Fernández Macías E, Hurley J, Vermeylen G. Fourth European Working Conditions Survey. 22-2-2007. European Foundation for the Improvement of Living and Working Conditions.

3. INSHT. VI ENCUESTA NACIONAL DE CONDICIONES DE TRABAJO. 2008. Ministerio de Trabajo y Asuntos Sociales.

4. Ministerio de Trabajo y Asuntos Sociales. REAL DECRETO 286/2006, de 10 de marzo, sobre la protección de la salud y la seguridad de los trabajadores contra los riesgos relacionados con la exposición al ruido. 2006.

5. Instituto Sindical de Trabajo Ambiente y SaIud. Especialidad de higiene industrial del plan de formación 2006 para empleados de la sanidad pública. 2006. CC.OO. federación de sanidad y sectores socio sanitarios.

6. Smith AP, Broadbent DE. Non-auditory effects of noise at work: A review of the literature. HSE, editor. 30/1991. 1991.

7. Murillo IC. [How does noise affect us? In our health, life styles and environs]. Rev Enferm 2007; 30(10):13-20.

8. Concha-Barrientos M C-LDSK. Occupational noise: assessing the burden of disease from work_related hearing impairment at national and local levels. 2004. Geneve, World Health Organization. (WHO Environmental Burden of Disease Series, $\left.\mathrm{N}^{\circ} .9\right)$.

9. World Health Organization. WHO Statistical Information System (WHOSIS). http://www.who.int/whosis/ en/. 2009. 1-4-2009.

10. Instituto Nacional de Estadistica. Defunciones según causa de muerte.INEbase. http://www.ine.es/inebmenu/ mnu_salud.htm\#2. 2009. 1-4-2009.

11. Bjorntorp P. Stress and cardiovascular disease. Acta Physiol Scand Suppl 1997; 640:144-148.

12. Saha S, Gandhi A, Das S, Kaur P, Singh SH. Effect of noise stress on some cardiovascular parameters and audiovisual reaction time. Indian J Physiol Pharmacol 1996; 40(1):35-40. 
13. de Hollander A, van Kempen EEMM, Houthuijs DJM, van Kamp I, Hoogenven RT, Staatseen BAM. Environmental noise: an approach for estimating health impacts at national and local level. Geneve, World Health Organization (Environmental Burden of Disease Series). In press.

14. Fogari R, Zoppi A, Vanasia A, Marasi G, Villa G. Occupational noise exposure and blood pressure. J Hypertens 1994; 12(4):475-479.

15. Butler MP, Graveling RA. NON-AUDITORY EFFECTS OF NOISE AT WORK: A CRITICAL REVIEW OF THE LITERATURE POST 1988. HSE Books, 1999.

16. Scottish Intercollegiate Guidelines Network. SIGN 50: A guideline developer's handbook. http://www.sign. ac.uk. 2008

17. von Elm E, Altman DG, Egger M, Pocock SJ, Gotzsche PC, Vandenbroucke JP. [The Strengthening the Reporting of Observational Studies in Epidemiology (STROBE) statement: guidelines for reporting observational studies]. Rev Esp Salud Pública 2008; 82(3):251-259.

18. Oxman AD, Cook DJ, Guyatt GH. Users' guides to the medical literature. VI. How to use an overview. Evidence-Based Medicine Working Group. JAMA 1994; 272(17):1367-1371

19. McBride P. Another view on overviews. JAMA 1995; 274(3):217-218.

20. Oxman AD, Sackett DL, Guyatt GH. Users' guides to the medical literature. I. How to get started. The Evidence-Based Medicine Working Group. JAMA 1993; 270(17):2093-2095

21. Jaeschke R, Guyatt GH, Sackett DL. Users' guides to the medical literature. III. How to use an article about a diagnostic test. $\mathrm{B}$. What are the results and will they help me in caring for my patients? The EvidenceBased Medicine Working Group. JAMA 1994; 271(9):703-707.

22. Laupacis A, Wells G, Richardson WS, Tugwell P. Users' guides to the medical literature. V. How to use an article about prognosis. Evidence-Based Medicine Working Group. JAMA 1994; 272(3):234-237.

23. Guyatt GH, Sackett DL, Cook DJ. Users' guides to the medical literature. II. How to use an article about therapy or prevention. A. Are the results of the study valid? Evidence-Based Medicine Working Group. JAMA 1993; $270(21): 2598-2601$

24. Virkkunen H, Kauppinen T, Tenkanen L. Long-term effect of occupational noise on the risk of coronary heart disease. Scand J Work Environ Health 2005; 31(4):291-299.

25. Virkkunen H, Harma M, Kauppinen T, Tenkanen L. The triad of shift work, occupational noise, and physical workload and risk of coronary heart disease. Occup Environ Med 2006; 63(6):378-386.

26. Virkkunen H, Harma M, Kauppinen T, Tenkanen L. Shift work, occupational noise and physical workload with ensuing development of blood pressure and their joint effect on the risk of coronary heart disease. Scand J Work Environ Health 2007; 33(6):425-434.

27. Sbihi H, Davies HW, Demers PA. Hypertension in noise-exposed sawmill workers: a cohort study. Occup Environ Med 2008; 65(9):643-646.

28. Davies HW, Teschke K, Kennedy SM, Hodgson MR, Hertzman C, Demers PA. Occupational exposure to noise and mortality from acute myocardial infarction. Epidemiology 2005; 16(1):25-32.

29. Melamed S, Fried Y, Froom P. The interactive effect of chronic exposure to noise and job complexity on changes in blood pressure and job satisfaction: a longitudinal study of industrial employees. J Occup Health Psychol 2001; 6(3):182-195.

30. Fujino Y, Iso H, Tamakoshi A. A prospective cohort study of perceived noise exposure at work and cerebrovascular diseases among male workers in Japan. J Occup Health 2007; 49(5):382-388.

31. McNamee R, Burgess G, Dippnall WM, Cherry N. Occupational noise exposure and ischaemic heart disease mortality. Occup Environ Med 2006; 63(12):813-819.

32. Willich SN, Wegscheider K, Stallmann M, Keil T. Noise burden and the risk of myocardial infarction. Eur Heart J 2006; 27(3):276-282.

33. Kristal-Boneh E, Melamed S, Harari G, Green MS. Acute and chronic effects of noise exposure on blood pressure and heart rate among industrial employees: the Cordis Study. Arch Environ Health 1995; 50(4):298-304.

34. Melamed S, Froom P, Kristal-Boneh E, Gofer D, Ribak J. Industrial noise exposure, noise annoyance, and serum lipid levels in blue-collar workers--the CORDIS Study. Arch Environ Health 1997; 52(4):292-298

35. Talbott EO, Gibson LB, Burks A, Engberg R, McHugh KP. Evidence for a dose-response relationship between occupational noise and blood pressure. Arch Environ Health 1999; 54(2):71-78.

36. Tomei F, Fantini S, Tomao E, Baccolo TP, Rosati MV. Hypertension and chronic exposure to noise. Arch Environ Health 2000; 55(5):319-325.

37. Fogari R, Zoppi A, Corradi L, Marasi G, Vanasia A, Zanchetti A. Transient but not sustained blood pressure increments by occupational noise. An ambulatory blood pressure measurement study. J Hypertens 2001; 19(6):1021-1027. 
38. Powazka EE. A cross-sectional study of occupational noise exposure and blood pressure in steelworkers. Noise Health 2003; 5(17):15-22.

39. Lusk SL, Hagerty BM, Gillespie B, Caruso CC. Chronic effects of workplace noise on blood pressure and heart rate. Arch Environ Health 2002; 57(4):273-281.

40. Lusk SL, Gillespie B, Hagerty BM, Ziemba RA. Acute effects of noise on blood pressure and heart rate. Arch Environ Health 2004; 59(8):392-399.

41. Jing Yuan, Miao Yang, Huiling Yao, Jianru Zheng, Qiaoling Yang, Sheng Chen et al. Plasma antibodies to heat shock protein 60 and heat shock protein 70 are associated with increased risk of electrocardiograph abnormalities in automobile workers exposed to noise. Cell Stress \& Chaperones 2005; 10(2):126-135.

42. Inoue M, Laskar MS, Harada N. Cross-sectional study on occupational noise and hypertension in the workplace. Arch Environ Occup Health 2005; 60(2):106-110.

43. Chang TY, Su TC, Lin SY, Jain RM, Chan CC. Effects of occupational noise exposure on 24-hour ambulatory vascular properties in male workers. Environ Health Perspect 2007; 115(11):1660-1664.

44. Chang TY, Jain RM, Wang CS, Chan CC. Effects of occupational noise exposure on blood pressure. J Occup Environ Med 2003; 45(12):1289-1296.

45. Ni CH, Chen ZY, Zhou Y, Zhou JW, Pan JJ, Liu N et al. Associations of blood pressure and arterial compliance with occupational noise exposure in female workers of textile mill. Chin Med J (Engl ) 2007; 120(15):1309-1313.

46. van Kempen EE, Kruize H, Boshuizen HC, Ameling CB, Staatsen BA, de Hollander AE. The association between noise exposure and blood pressure and ischemic heart disease: a meta-analysis. Environ Health Perspect 2002; 110(3):307-317

47. Duncan R, Easterly CE, Griffith J, Aldrich TE. The effect of chronic environmental noise on the rate of hypertension: a meta_analysis. Environ Int 1993; 19:359-369.

48. Holand S, Girard A, Laude D, Meyer-Bisch C, Elghozi JL. Effects of an auditory startle stimulus on blood pressure and heart rate in humans. J Hypertens 1999; 17(12 Pt 2):1893-1897.

49. Melamed S, Ben Avi I, Luz J, Green MS. Repetitive work, work underload and coronary heart disease risk factors among blue-collar workers- the CORDIS study. Cardiovascular Occupational Risk Factors Determination in Israel. J Psychosom Res 1995; 39(1):19-29.

50. Theorell T. Family history of hypertension--an individual trait interacting with spontaneously occurring job stressors. Scand J Work Environ Health 1990; 16 Suppl 1:74-79.

51. Melamed S, Harari G, Green MS. Type A behavior, tension, and ambulatory cardiovascular reactivity in workers exposed to noise stress. Psychosom Med 1993; 55(2):185-192.

52. Tomei C, Abraini JH, Rostain JC. A new device for behavioral analysis on rats exposed to high pressure. Physiol Behav 1991; 49(2):393-396.

53. Umemura M, Honda K, Kikuchi Y. Influence of noise on heart rate and quantity of work in mental work. Ann Physiol Anthropol 1992; 11(5):523-532.

54. Lesnik H, Makowiec-Dabrowska T. Hemodynamic reactions to monotonous work performed in silence and in noise of $70 \mathrm{~dB}$ (A). Pol J Occup Med 1989; 2(1):51-61.

55. De Boer SF, Van der GJ, Slangen JL. Plasma catecholamine and corticosterone responses to predictable and unpredictable noise stress in rats. Physiol Behav 1989; 45(4):789-795.

56. Parrot J, Petiot JC, Lobreau JP, Smolik HJ. Cardiovascular effects of impulse noise, road traffic noise, and intermittent pink noise at LAeq $=75 \mathrm{~dB}$, as a function of sex, age, and level of anxiety: a comparative study. I. Heart rate data. Int Arch Occup Environ Health 1992; 63(7):477-484.

57. Kristensen TS. Cardiovascular diseases and the work environment. A critical review of the epidemiologic literature on nonchemical factors. Scand J Work Environ Health 1989; 15(3):165-179.

58. Case Control study regarding Myocardial infarction, shiftwork and occupational exposure to noise. Proceedings of the 9th International Symposium on Epidemiology in Occupational Health.; 92 Sep 23; National Institute for Occupational Safety and Health, 1992.

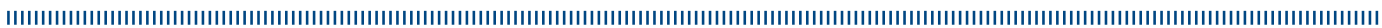

